\title{
Cure of hyperparathyroidism in pregnancy by sternotomy and removal of a mediastinal parathyroid adenoma
}

\author{
D P Rooney, A I Traub, C F J Russell, D R Hadden
}

\begin{abstract}
Summary
Primary hyperparathyroidism is rarely reported during pregnancy but can cause significant maternal and neonatal morbidity. We report a case of hyperparathyroidism during pregnancy requiring median sternotomy for resection of a mediastinal parathyroid adenoma. Surgery resulted in normalisation of serum calcium, resolution of symptoms, and prevented neonatal hypocalcaemia.
\end{abstract}

Keywords: hyperparathyroidism; mediastinal adenoma; pregnancy

Primary hyperparathyroidism is infrequently reported during pregnancy. The incidence of hyperparathyroidism in women of childbearing age is approximately eight cases per 100000 per year, yet in a recent review of the literature only 109 cases during pregnancy were reported between 1930 and $1990 .{ }^{1}$ Untreated hyperparathyroidism may have serious consequences for both mother and child and surgical removal of the parathyroid tumour during pregnancy may be required. Identification and removal of a hyperfunctioning parathyroid tumour, including most mediastinal tumours, can usually be accomplished by cervical exploration. ${ }^{2}$ Occasionally, because of their deep location in the chest, median sternotomy is necessary.

\section{Case report}

A 31-year-old woman presented in August 1995 with abdominal pain. Serum calcium was $2.70 \mathrm{mmol} / 1$ (normal range $2.10-2.57 \mathrm{mmol} / \mathrm{l}$ ) and serum parathormone $60 \mathrm{pg} / \mathrm{ml}(10-50$ $\mathrm{pg} / \mathrm{ml}$ ). There was no family history of hypercalcaemia. Four weeks later the patient discovered that she was pregnant and further investigation was deferred. In November 1995 (at 14 weeks gestation) she was admitted to hospital with nausea, vomiting and abdominal pain. Serum calcium had increased to 2.93 $\mathrm{mmol} / \mathrm{l}$, phosphate was $0.96 \mathrm{mmol} / \mathrm{l}(0.8-1.55$ $\mathrm{mmol} / \mathrm{l})$ and alkaline phosphatase $52 \mathrm{U} / \mathrm{l}$ (35-120 U/1). Urinary calcium excretion was elevated, 18.8 and $20.0 \mathrm{mmol} / 24 \mathrm{~h}$ on two occasions (3.0-9.0). Serum parathormone remained inappropriately elevated at $38 \mathrm{pg} / \mathrm{ml}$. Serum immunoglobulin electrophoresis pattern, angiotensin-converting enzyme and amy- lase were normal. No abnormality was identified on chest radiography.

Following rehydration, serum calcium fell to $2.70 \mathrm{mmol} / 1$ and symptoms resolved. Cervical exploration was then carried out. Normal left superior and left inferior parathyroids were identified. No parathyroid tissue was identified on the right side. Total right thyroid lobectomy was performed but no intrathyroid parathyroid tumour was found. Postoperatively, serum calcium remained elevated at $2.80 \mathrm{mmol} / 1$ and parathormone was $33 \mathrm{pg} / \mathrm{ml}$. At 18 weeks and 20 weeks gestation further admission to hospital was necessary because of nausea, vomiting and abdominal discomfort. Total serum calcium had not risen further (2.84 and 2.62 $\mathrm{mmol} / \mathrm{l}$, respectively) but on both occasions symptoms improved rapidly following rehydration.

Magnetic resonance imaging (MRI) of the chest suggested a small low-signal structure within the thymus but was unable to identify the lesion as a parathyroid adenoma. To obtain better resolution, a computed tomography (CT) scan with dynamic enhancement of the mediastinum was performed using appropriate abdominal shielding. A lesion of less than $1 \mathrm{~cm}$ was identified in front of the aortic arch, within the thymus (figures 1 and 2). The enhancement pattern was consistent with a parathyroid adenoma. Because of its deep intrathoracic location, the tumour was judged inaccessible via a cervical approach. Median sternotomy, mediastinal exploration and total thymectomy were therefore carried out at 23 weeks gestation. Dissection of the left thymic lobe revealed a 5-mm diameter (480 $\mathrm{mg}$ ) parathyroid adenoma. Postoperatively, serum calcium immediately fell to $2.2 \mathrm{mmol} / \mathrm{l}$ and has subsequently remained normal. She gave birth at 38 weeks gestation by normal delivery to a healthy boy weighing $3.2 \mathrm{~kg}$. There was no neonatal hypocalcaemia.

\section{Discussion}

Total serum calcium normally declines as pregnancy progresses, ${ }^{3}$ whereas ionised calcium and parathormone appear to change little. ${ }^{4}$ During pregnancy the placenta actively transports calcium ions to the foetus but does not allow transfer of parathormone. ${ }^{5}$ Maternal hyperparathyroidism can therefore result in foetal hypercalcaemia which substantially increases the risk of spontaneous abortion. ${ }^{6}$ 
Figure 1 A dynamically enhanced CT scan of the anterior mediastinum identifying an intrathymic parathyroid adenoma

Figure 2 Intrathymic parathyroid $\begin{array}{r}\text { Intrathymic } \\ \text { adenoma }\end{array}$ position demonstrated by a longitudinally re-formatted CT scan of anterior mediastinum

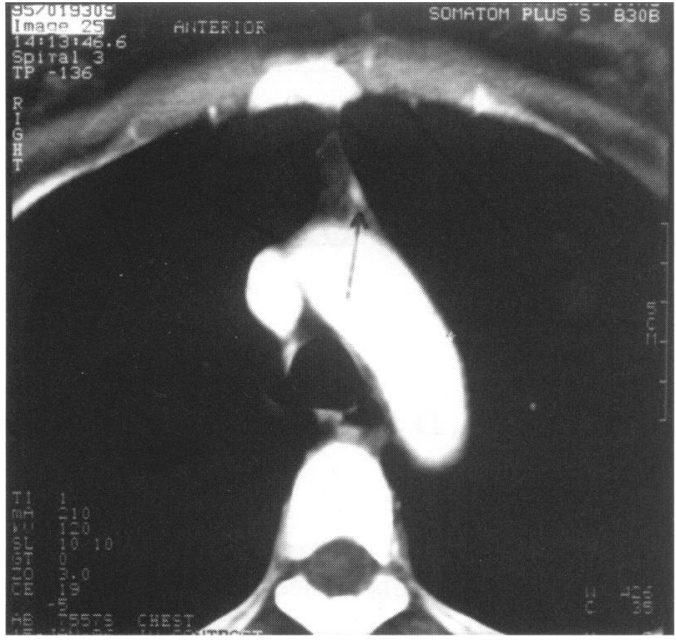

\begin{tabular}{|l|}
\hline Learning points \\
\hline hyperparathyroidism during pregnancy can cause \\
significant medical problems for both mother \\
and child \\
total serum calcium normally falls as pregnancy \\
progresses so that hypercalcaemic symptoms may \\
be experienced at lower total serum calcium \\
levels than usual \\
in hyperparathyroidism, parathormone is not \\
always increased but should be inappropriately \\
high for the serum calcium concentration \\
a helical CT scan is helpful in mediastinal \\
parathyroid adenoma localisation, especially \\
during pregnancy when radioisotope techniques \\
are contraindicated \\
parathyroidectomy during the second trimester \\
of pregnancy effectively prevents neonatal \\
hypocalcaemia
\end{tabular}

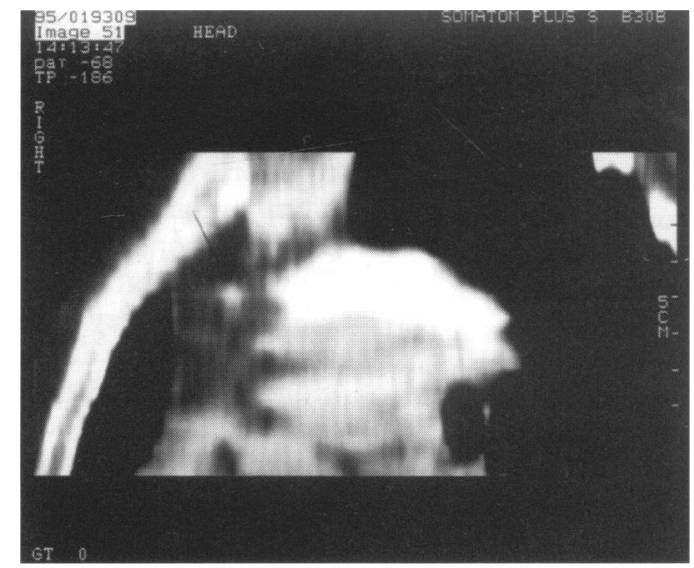

Conversely, after delivery, when the maternal transplacental supply of calcium ceases, neonatal hypocalcaemia becomes the major problem. This may occur because the neonate is unable to mobilise calcium stores adequately as a result of prolonged parathyroid gland suppression.

Up to $40 \%$ of inferior parathyroid glands lie within the thymus gland, ${ }^{7}$ and as most are located in the cervical tongue of thymus, they are surgically accessible from the neck. Overall, less than $2 \%$ of parathyroid adenomas require sternotomy for removal and approximately $50 \%$ of these will be intrathymic. ${ }^{8}$ Our patient is the first reported case of hyperparathyroidism during pregnancy who required me-

1 Kelly TR. Primary hyperparathyroidism during pregnancy Surgery 1991;110:1028-34

2 Nathaniels EK, Nathaniels AM, Wang C. Mediastinal parathyroid tumors: a clinical and pathological study of 84 cases. Ann Surg 1970;171:165-70.

3 Mimouni F, Tsang RC, Hertzberg V, Neumann V, Ellis K Parathyroid hormone and calcitriol changes in normal and insulin dependent diabetic pregnancies. Obstet Gynecol 1989;74:49-54.

4 Davis OK, Hawkins DS, Rubin LP, Posillico JT, Brown EM, Schiff I. Serum parathyroid hormone (PTH) in pregnant women determined by an immunoradiometric assay for intact PTH. ₹ Clin Endocrinol Metab 1988;67:850-2.

5 Pitkin RM, Reynolds WA, Williams GA, Hargis GK Calcium metabolism in normal pregnancy: longitudinal Calcium metabolism in normal pregnancy:
study. Am f Obstet Gynecol 1979;133:781-90. dian sternotomy for successful retrieval of the hyperfunctioning tumour. Some difficulty was encountered in localising the adenoma because radioisotope techniques are contraindicated during pregnancy. Ultrasonography has proved useful in identifying parathyroid adenomas in the neck ${ }^{9}$ but cannot localise tumours within the mediastinum. Experience with MRI is still limited and although the presence of an intrathymic lesion was suggested using this technique, it could not be confirmed as a parathyroid tumour. Improved resolution was achieved by means of a short CT scan with dynamic (helical) enhancement.

Retrospective case reviews suggest that foetal and neonatal complications are more common in medically managed cases and that maternal morbidity from neck exploration surgery is low. ${ }^{1}$ In particular, successful surgery eliminates the risk of neonatal hypocalcaemia. Median sternotomy to remove a mediastinal parathyroid adenoma will tend to be associated with higher morbidity than cervical exploration. In a series of 38 patients from the Mayo Clinic who underwent median sternotomy for removal of a parathyroid adenoma, $21 \%$ had postoperative chest complications. ${ }^{8}$ Although our patient subsequently developed a pulmonary embolus, it was felt that in the presence of persisting hypercalcaemia and significant symptoms, formal mediastinal exploration was warranted.

6 Ludwig GD. Hyperparathyroidism in relation to pregnancy. $N$ Engl f Med 1962;267:637-42.

7 Wang C. The anatomic basis of parathyroid surgery. Ann Surg 1976;183:271-5.

8 Russell CFJ, Edis AJ, Scholz DA, Sheedy PF, Van Heerden JA. Mediastinal parathyroid tumors. Experience with 38 tumors requiring mediastinotomy for removal. Ann Surg tumors requiring

9 Sauer M, Steere A, Parsons MT. Hyperparathyroidism in pregnancy with sonographic documentation of a parathyroid adenoma: a case report. $\mathcal{F}$ Reprod Med 1985;30: 615-7. 